See Article page 1131.

\section{Commentary: Deus ex machina: Bad coding or perfect plot device?}

\author{
Hillary Lia, BComp, Derrick Y. Tam, MD, PhD, and \\ Stephen E. Fremes, MD, MSc
}

The number of machine-learning (ML) publications in the medical sciences has increased dramatically in the last decade. ${ }^{1}$ The recent boom in ML models has led to awe-inspiring promises about the potential for these technologies to improve outcomes for patients by making earlier diagnoses, selecting better treatments, and making more accurate risk stratifications than humans. Impressive results have been published for detecting arrhythmias from electrocardiograms with better sensitivity and specificity than individual cardiologists, ${ }^{2}$ predicting postoperative liver dysfunction following aortic arch surgery, ${ }^{3}$ artificial intelligence-enhanced robotic surgery, ${ }^{4}$ early detection of intraoperative hypoxemia, ${ }^{5}$ and a plethora of other clinical applications. Despite the promise of ML techniques, this technology is not without its pitfalls. Although ML techniques are often assumed to be superior to traditional statistics, these techniques exist on a spectrum. In fact, there exist many cases in which ML is shown to have no evidence of being superior to logistic regression. ${ }^{6}$ ML applications require far more data to avoid overfitting ${ }^{7}$ compared with traditional statistics. Thus, an ML approach is appropriate only in select circumstances. Furthermore, it is not uncommon for models that performed well during training and testing to underperform when exposed to the complexity of real-world application. ${ }^{8}$ In addition, ML models are largely nonexplainable in that there is little indication for how the model comes to a prediction. As promising ML models continue to emerge as clinical tools, clinicians require the knowledge and skills to accurately

\footnotetext{
From the Division of Cardiac Surgery, Department of Surgery, Schulich Heart Centre, Sunnybrook Health Sciences Centre, University of Toronto, Toronto, Ontario, Canada.

Disclosures: The authors reported no conflicts of interest.

The Journal policy requires editors and reviewers to disclose conflicts of interest and to decline handling or reviewing manuscripts for which they may have a conflict of interest. The editors and reviewers of this article have no conflicts of interest.

Received for publication March 31, 2021; revisions received March 31, 2021; accepted for publication March 31, 2021; available ahead of print April 16, 2021.

Address for reprints: Stephen E. Fremes, MD, MSc, Schulich Heart Centre, Sunnybrook Health Sciences Centre, 2075 Bayview Ave, Room H4 05, Toronto, Ontario, M4N 3M5 Canada (E-mail: stephen.fremes@sunnybrook.ca).

J Thorac Cardiovasc Surg 2022;163:1138-9

$0022-5223 / \$ 36.00$

Copyright (c) 2021 by The American Association for Thoracic Surgery

https://doi.org/10.1016/j.jtcvs.2021.03.112
}

Check for updates

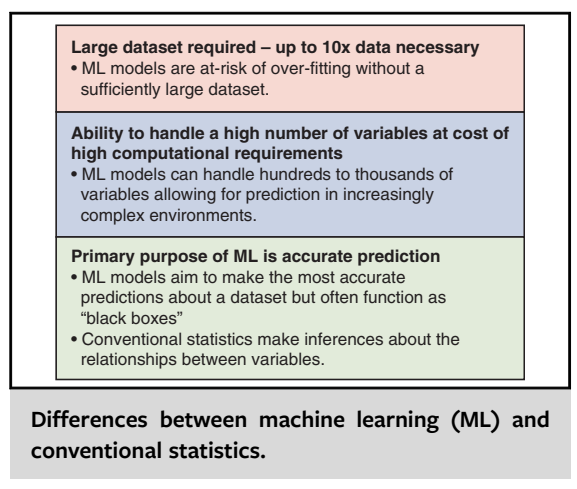

CENTRAL MESSAGE

Machine learning, when applied

correctly, can be a powerful tool

to solve complex statistical

problems and improve our sta-

tistical models.

interpret results and assess potential pitfalls before implementation in clinical practice.

In this issue of the Journal, Domaratzki and Kidane ${ }^{9}$ summarize ML for clinicians, including indications for implementation, basic concepts, interpretation, and most commonly used algorithms. The authors' witty title includes deus ex machina, Latin for a "god from a machine," and a reference to plot devices with origins in ancient Greek theater whereby an almighty powerful deity rescues our protagonist from a hopeless situation. ${ }^{10}$ This reference suggests that ML may be that all-powerful tool that rescues us from all our statistical obstacles, but the authors correctly assert that without a concrete understanding of the techniques and pitfalls of ML, there is significant potential for improper use and misguided interpretation of results. The authors aim to mitigate this risk by providing readers with a concise review of need-to-know concepts focusing on a division of ML known as supervised learning. Supervised learning models learn to predict outcomes from a dataset where the outcome of interest is initially labeled by human experts, providing the model with a "ground truth" that allows for measures of model performance and accuracy. There are several models that are selected, using considerations such as computational capacity, dataset size, and number of features (Figure 1). In ML terminology, individual records within a dataset are labeled as vectors. Methods are evaluated by splitting the dataset into a training set and test set, as in conventional statistical modeling. An abundance of caution should be exercised in ML models where 


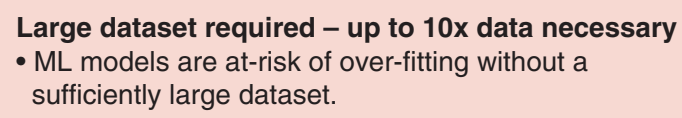

Ability to handle a high number of variables at cost of high computational requirements

- ML models can handle hundreds to thousands of variables allowing for prediction in increasingly complex environments.

Primary purpose of ML is accurate prediction

- ML models aim to make the most accurate predictions about a dataset but often function as "black boxes"

- Conventional statistics make inferences about the relationships between variables.

FIGURE 1. Differences between machine learning (ML) and conventional statistics.

cross-validation is used for small datasets, as there is an excessively increased risk of overfitting. The authors introduce feature importance tools as means to estimate which variables are most important for prediction by the model.

Domaratzki and Kidane expertly and concisely summarize key concepts for competent interpretation of ML literature. As the push for ML education in medical training grows, ${ }^{11,12}$ resources for clinicians to learn relevant skills for interpretation become increasingly important. The authors peel back the curtain on ML for clinicians to begin taking their role as active and thoughtful participants. In the coming years, ML will continue to grow and integrate into clinical environments. Clinicians who are wellinformed ensure that ML tools are used in a manner that is safe and appropriate for patients. Circling back to the authors' reference of deus ex machina, its use in literature and theater may be classified as "lazy writing" and an easy "cop-out" to save the protagonist from their troubles. ${ }^{10}$ However, this trope, when used appropriately, can also create an elegant ending to a well-written story. The same can be said about ML; when applied correctly, its power can be harnessed to improve statistical models and better patient outcomes. As such, this review by Domaratzki and Kidane is more relevant than ever to our readership as we begin to better understand ML and its appropriate place in our arsenal of statistical techniques.

\section{References}

1. Meskó B, Görög M. A short guide for medical professionals in the era of artificial intelligence. NPJ Digit Med. 2020;3:126.

2. Rajpurkar P, Hannun AY, Haghpanahi M, Bourn C, Ng AY. Cardiologist-level arrhythmia detection with convolutional neural networksar. arXiv. 2017;1707. 01836.

3. Lei G, Yang L, Zhang C, Fang Z, Li J, Wang G. Using machine learning to predict postoperative liver dysfunction after aortic arch surgery. J Cardiothorac Vasc Anesth. February 20, 2021 [Epub ahead of print].

4. Gehlbach PL. Robotic surgery for the eye. Nat Biomed Eng. 2018;2:627-8.

5. Lundberg SM, Nair B, Vavilala MS, Horibe M, Eisses MJ, Adams T, et al. Explainable machine-learning predictions for the prevention of hypoxaemia during surgery. Nat Biomed Eng. 2018;2:749-60.

6. Christodoulou E, Ma J, Collins GS, Steyerberg EW, Verbakel JY, Van Calster B, et al. A systematic review shows no performance benefit of machine learning over logistic regression for clinical prediction models. J Clin Epidemiol. 2019; 110:12-22.

7. Van Der Ploeg T, Austin PC, Steyerberg EW. Modern modelling techniques are data hungry: a simulation study for predicting dichotomous endpoints. BMC Med Res Methodol. 2014;14:137.

8. Topol EJ. High-performance medicine: the convergence of human and artificial intelligence. Nat Med. 2019;25:44-56.

9. Domaratzki M, Kidane B. Deus ex machina? Demystifying rather than deifying machine learning. J Thorac Cardiovasc Surg. 2022;163:1131-7.e4.

10. Maio A. Deus ex machina-meaning, definition \& examples in movies; 2020 Available at: https://www.studiobinder.com/blog/deus-ex-machina-meaningdefinition/. Accessed March 26, 2021.

11. McCoy LG, Nagaraj S, Morgado F, Harish V, Das S, Celi LA. What do medica students actually need to know about artificial intelligence? NPJ Digit Med. 2020;3:86.

12. Pucchio A, Eisenhauer EA, Moraes FY. Medical students need artificial intelligence and machine learning training. Nat Biotechnol. 2021;39: 388-9. 